Voix et Images

voixetimages

\title{
Le Matou d'Yves Beauchemin : du fait littéraire à la chaîne de productions-médias
}

\section{Catherine Saouter}

Volume 12, numéro 3 (36), printemps 1987

Yves Beauchemin

URI : https://id.erudit.org/iderudit/200654ar

DOI : https://doi.org/10.7202/200654ar

Aller au sommaire du numéro

Éditeur(s)

Université du Québec à Montréal

ISSN

0318-9201 (imprimé)

1705-933X (numérique)

Découvrir la revue

Citer cet article

Saouter, C. (1987). Le Matou d’Yves Beauchemin : du fait littéraire à la chaîne de productions-médias. Voix et Images, 12(3), 393-403.

https://doi.org/10.7202/200654ar d'utilisation que vous pouvez consulter en ligne.

https://apropos.erudit.org/fr/usagers/politique-dutilisation/ 


\title{
Le Matou d'Yves Beauchemin: du fait littéraire à la chaîne de productions-médias
}

\author{
par Catherine Saouter, Université du Québec à Montréal
}

La présente étude veut rendre compte d'un phénomène courant de la culture de masse à travers un exemple particulier; celui de la chaîne de productions culturelles occasionnée par le roman d'Yves Beauchemin le Matou!.

Un best-seller, une édition de poche, un film, une télé-série, deux couvertures de livre, une affiche, des articles de presse écrite, etc. participent à l'exploitation d'une histoire que tout le monde connait et qui raconte comment «Florent obtient un restaurant». Qu'est-ce que ce récit a de si particulier pour se prêter à tant de ressources et connaître une telle longévité? Quelques éléments de réponse seront ici proposés. Deux fils d'Ariane ont été utilisés pour effectuer la traversée des différents textes: d'une part, l'observation des techniques de nomination des personnages ${ }^{2}$; d'autre part, celle des techniques de construction des lieux. Ces deux approches ont pour but de retracer certains enjeux idéologiques qui se manifestent tout au long de l'exploitation du succès du Matou, en tant que texte et en tant que livre.

\section{Le Roman}

Le texte du Matou débute avec le nom d'un personnage: Médéric Duchêne. Quelle que soit la réceptivité du lecteur, ce nom-là ne manque pas de surprendre un peu. Ce Médéric Duchêne, de surcroît, trépasse au cours de la première phrase, ce qui peut également surprendre. Le Matou n'est pas un roman policier et dispose, de toute évidence, d'autres ressources que ce personnage, puisqu'il compte cinq cent quatre-vingt-trois pages. C'est de cet étonnement que vient la décision d'analyser les stratégies de l'auteur concernant les noms de ses personnages et leur mode d'introduction. S'agissant d'une relecture, cette analyse porte seulement sur les quatre-vingt-treize premières pages, de l'entrée de Médéric Duchêne à celle de Monsieur Émile qui a l'importance que l'on sait.

De façon remarquable, l'auteur livre, dépendamment du personnage, un nom et un prénom, un statut social, un statut familial et amical, des éléments biographiques, ou, abruptement, un simple prénom. On peut effectuer un premier classement des personnages en six catégories:

1 - ceux dont on nous livre, à la première désignation, un nom et un prénom: Médéric Duchêne, Rosario Gladu, Gustave Bleau, Albert N. Paquette, Florent Boissonneault;

2 - ceux dont on nous précise un titre renseignant sur le statut social: monsieur Spufferberg, Capitaine Galarneau, le notaire Philippe Paimparé; 
3 - ceux qui sont désignés par leurs caractéristiques sociales ou pittoresques: les secrétaires, les clients;

4 - ceux dont l'introduction du nom est précédée par une mise en scène préalable: Egon Ratablavasky, l'abbé Jeunehomme, monsieur Émile;

5 - ceux dont il est immédiatement établi un lien avec un autre personnage: son ami Aurélien Piquot, son collègue Slipskin;

' 6 - ceux qui ne possèdent qu'un prénom: Élise, Ange-Albert, Bertrand.

Ces catégories livrent des renseignements sur les rôles structuraux du récit et sur son univers référentiel.

Ce dernier est particulièrement perceptible grâce à la deuxième catégorie. Mademoiselle Rachel est femme de chambre, Le Capitaine Galarneau est militaire et/ou marin. Monsieur Spufferberg est chef d'une petite entreprise, etc. Le qualificatif "petit» de le petit José Biondi décrit peut-être une petite taille, mais dans ce contexte, il est inévitablement perçu comme une marque de sa position inférieure d'aide-cuisinier. On a appris, par ailleurs, qu'Egon Ratablavasky était grand (p. 11).

Les personnages possédant seulement un prénom sont typés. Gardons en réserve pour le moment le cas de Florent et considérons Élise, immédiatement énoncée comme épouse du précédent, dont le seul désir est d'avoir des enfants; Ange-Albert, le drop-out type, indifférent aux ambitions du milieu des affaires (p. 43); Bertrand, l'homosexuel, entouré de nombreuses allusions douteuses sur ce type de sexualité (p. 13, 16, 23, 27, 60,91); Gisèle, propriété exclusive de son partenaire (p. 90-91).

Les personnages féminins sont suffisamment peu nombreux pour qu'on les considère un à un. D'Élise et de Gisèle on a déjà dit leur appartenance à deux hommes, laquelle est reconnue par le groupe environnant. Mademoiselle Relique (sic) renvoie à la classe des travailleurs et, en tant que secrétaire, est présentée comme l'alter ego du patron. Floretta Chouinard et Rachel Gourdin, curieusement, ont une identité complète. Madame Chouinard est une mère célibataire ou abandonnée depuis longtemps par le père de son enfant; elle travaille dans un bar topless. Rachel Gourdin est employée dans un hôtel. Toutes les deux sont associées au monde de la prostitution.

Notons enfin que, mis à part le capitaine Galarneau et l'abbé Jeunehomme aux statuts sociaux de haut prestige, l'auteur fournit toujours des renseignements sur les activités sexuelles de chacun des personnages masculins.

Lorsqu'on procède à une récapitulation, on remarque que les personnages se situent par rapport à un axe - celui de la normalité? - : celui d'hommes au statut social conforme, rattaché aux institutions et au monde des affaires. 
Lorsque Monsieur Émile, dans un dialogue remarqué, revendique l'appellation monsieur Émile, il opère une nette transgression puisqu'il revendique à la fois sa place de marginal auprès d'Élise, Ange-Albert et autres Bertrand (les agents dits passifs de la société) et une place dans le monde des hommes (celui des agents dits actifs de la société). Tout au long du roman, la relation entre Florent et monsieur Émile entérine une certaine vision de la société: autant au plan de l'intimité, Florent aimera-t-il l'enfant, autant sur le plan social, accumulera-t-il les actes manqués qui auraient éventuellement sauvé l'enfant. Tout se passe comme si la marginalité était un poids mort de la société : composer avec elle serait une perte d'énergie, énergie que Florent, effectivement, ne trouve jamais le temps d'employer, si ce n'est dans le cas du chat (soins vétérinaires). Or il est aisé de retracer l'analogie faite entre ce chat et Egon Ratablavasky. Pour employer un terme greimassien, l'opposant de monsieur Émile est précisément Egon Ratablavasky, représentant type de la "normalité» (richesse, puissance, sexualité galopante). Comme on le sait, l'économie du roman repose sur les luttes symboliques de ces deux personnages et il s'achève sur leurs morts (effective ou présumée). Autour de ces deux champions, s'organisent les familles de protagonistes, les clans des bons et des méchants.

Au long de ces quatre-vingt-treize pages, les noms des personnages subissent diverses modifications jusqu'à parvenir à une formulation opérationnelle. Pour ne citer qu'un exemple, Florent est successivement appelé Florent Boissonneault, mon cher monsieur Florent, Bwassono, monsieur Boissonneault, boss et finalement, Florent. Pendant que certains accèdent à leur nom de route, d'autres disparaissent du texte. C'est le cas de la quasi totalité des personnages qui avaient une fonction avant tout référentielle. De façon symptomatique, le patron de Florent passe de monsieur Spufferberg à tu avant son éviction définitive. Les personnages qui poursuivent la traversée du roman sont répartis en fonction des paradigmes évoqués plus haut, dont ils restent des représentants synecdotiques. Les hommes sont appelés par leur nom de famille, les femmes et les hommes passifs par leurs prénoms, monsieur Émile reste monsieur Émile.

Reste à considérer Florent que la multiplicité des désignations ancre dans une réalité circonscrite. Le passage au prénom ne doit pas la faire oublier; ce prénom est le signe, en même temps, d'une vulnérabilité, d'une intimité et du statut du héros avec lequel le lecteur doit avoir une relation de promiscuité et de familiarité.

La lecture de ces quatre-vingt-treize pages permet, tout en découvrant les divers personnages, de comprendre leurs différentes quêtes et les médiations utilisées pour les réaliser. Or, au terme de ces pages, d'une façon ou d'une autre, ils sont tous arrivés à leurs fins: Florent a un restaurant, Élise a un enfant (d'adoption), Émile s'est fait prendre en charge, Gladu a un article pour son journal, etc. Restent quatre cents pages à occuper. L'auteur utilise deux ressources. La première consiste à faire basculer le récit du genre réaliste dans le fantastique: il ressuscite Egon Ratablavasky. La seconde, et non la 
moindre, vient de ce qu'un personnage n'a pas encore obtenu ce qu'il désirait: l'abbé Jeunehomme n'a pas encore trouvé le poêle, la truie de Gogol. Cet abbé Jeunehomme ressemble fort à un alter ego de Florent et il est l'instigateur d'un roman dans le roman. Cet aspect particulier du Matou mériterait d'être considéré en soi et ne peut être davantage développé ici.

La topographie sociale décrite dans les lignes qui précèdent est sise dans une géographie soigneusement construite par l'auteur. Toujours dans le même espace du roman, soit les quatre-vingt-treize premières pages, Yves Beauchemin déploie une mappemonde qui va des pyramides d'Égypte à Vancouver en passant par la France et de nombreux villages du Québec; au centre, Montréal, théâtre principal des actions et, dans Montréal, le Plateau MontRoyal.

Si on y regarde d'un peu plus près, il saute aux yeux que la méthode employée par Beauchemin pour évoquer ces lieux consiste à interpeller le savoir du lecteur suivant des axes bien définis. En effet, la lecture la plus superficielle du texte frappe par sa pauvreté en descriptions. Quoiqu'en ait dit la presse, il n'y a aucune similitude possible entre «le Montréal de Beauchemin" et "le Paris de Balzac», ainsi qu'on a pu le lire à diverses reprises.

L'auteur a d'abord et avant tout recours à la citation de repères topographiques (noms de rues, indications d'intersections, numéros de bâtiments). Plan de Montréal en mains, le lecteur peut aisément retrouver et visualiser les lieux cités. Ce qui est convoqué ici, c'est l'expérience du lecteur, acquise dans l'esprit de l'auteur, avec lequel il partage un même territoire. Pour le lecteur étranger, il y a là un effet d'exotisme par lequel «les coins Marquette et Papineau» résonnent comme les "Médéric Duchêne» et les "Rosario Gladu».

L'auteur utilise aussi la figure de la métaphore. En comparant le poids des soucis à celui des pyramides ou le froid à celui du Pôle nord, l'auteur évoque, non pas une expérience qui témoigne d'un déplacement sur les lieux, mais un savoir du lecteur qui n'y est pas allé mais en a entendu parler. C'est littéralement l'exemple de l'article de Gladu qui parle de «la Venise du Québec».

La méthode peut aussi être celle du renseignement érudit. Essentiellement fourni par l'abbé Jeunehomme, il construit l'image de l'historien spécialisé, enfermé dans son discours, et persuadé que tout un chacun sait où se trouve Fontenay-les-Tours. Pour preuve de ce préjugé, il y a ce qualificatif «néogrecque" que l'auteur attribue à une façade. Le qualificatif aurait du être «néo-classique» et manifeste un emprunt erroné - volontaire ou involontaire - au glossaire de l'histoire de l'art.

Cette géographie est fortement ethno-centrée et l'impression se confirme lorsqu'on croise les Pirates de Pittsburgh qui, bien qu'américains, sont propriété de bien des Montréalais et des Québécois en général.

Cet univers centripète coïncide certainement de façon précise avec celui du lecteur moyen et il n'est pas douteux, qu'en matière de réception, la 
méthode d'Yves Beauchemin est efficace. Elle évite d'assommantes pages de lecture tout en prenant le soin d'évoquer une mappemonde suffisamment vaste pour donner un sentiment d'inscription dans le monde.

Il ressort de ce qui précède une adéquation manifeste entre l'univers du lecteur et celui de l'auteur. Les thématiques traitées à travers les actions de ce type de personnages dans ce type de lieux amèneraient à confirmer la justesse des traitements dans le roman par rapport à l'horizon d'attente: volonté de réussite des jeunes entrepreneurs, fatalité de l'échec, réduplication de l'image maternelle, réduplication du contexte social, etc. Cette coïncidence a sans doute joué dans l'effet best-seller dont l'ampleur a été telle qu'il a mis en route une chaîne de production d'objets culturels.

\section{Le Film}

Le phénomène de l'adaptation a l'intérêt de permettre aisément de retracer, par les distorsions imposées à l'œuvre de départ, des aspects de la vision du monde du nouveau producteur. Les journalistes ont tous souligné la "fidélité" de l'adaptation, certains même (Séquences no 122, p. 38) allant jusqu'à la qualifier de servile.

Cette servilité n'est qu'apparente. Certes, les personnages et les actions sont identiques. Mais, pour toutes sortes de raisons, Jean Beaudin et son équipe ont simplifié, tamisé la matière du roman pour la faire entrer dans le cadre des deux heures d'un film et des six heures d'une télé-série ${ }^{3}$. Il n'est pas inutile de savoir que les deux produits ont été tournés de concert avec un système de claquettes de couleur permettant d'identifier les séquences réservées au film, à la télé-série ou communes aux deux. Deux possibilités se présentent : - soit que les deux produits forment un ensemble homogène, ce qui, dans l'analyse pour l'un, vaut pour l'autre: - soit qu'apparaissent à la lecture d'importantes différences. En fait, le visionnement confirme la première hypothèse.

Le casting de Jean Beaudin fait tomber de nombreuses têtes. La sélection est fonction d'un paradigme de la famille. Famille comme terme sociologique: parents, enfants, époux, épouse, etc.; famille comme terme structural: clan des bons et clans des méchants. Dans le premier cas, cela signifie que l'on verra à de fréquentes reprises le père et la mère de Florent, la femme du maire, la gardienne d'Émile, etc. Dans le deuxième cas, Beaudin procède par condensation. Par exemple, il ramène à un seul personnage les différentes maîtresses d'Ange-Albert: l'unique Rosine devient du même coup une bellesœur. Ou encore, il supprime le Capitaine Galarneau et conserve Rachel Gourdin. Cela lui permet de consacrer un temps inconsidéré à la scène de ménage entre Florent et Élise dans laquelle l'épouse défend sauvagement l'ordre familial menacé.

Il est évident qu'aussi conservatrice soit-elle, la problématique de Beauchemin sur le pouvoir financier a été aplatie par Beaudin au profit d'une apologie nostalgique de la famille. 
Le traitement des lieux est tout autant symptomatique. Suivant les mêmes critères de lecture que pour le roman, de la première scène à l'entrée de monsieur Émile, on compte treize extérieurs qui durent en moyenne vingt secondes. Certains durent à peine dix secondes, le deuxième dure deux minutes, le douzième cinquante secondes.

Les deux premières séquences offrent un point de vue qui suppose un travail relativement savant et acrobatique. Ces deux séquences ont un contenu distinct, l'une étant consacrée à la mise en contexte, la seconde étant le départ de la narration proprement dite. En fait, les effets de plongée et de contreplongée disparaissent très vite et les quelques utilisations ultérieures deviennent fonction d'un usage banalisé au cinéma (regard subjectif, effet cartepostale, regard de Dieu). La caméra prend très vite une position frontale et opère de réguliers travellings latéraux ou légèrement de biais. Le champ est littéralement un théâtre pour les personnages: de façon récurrente, ils entrent par un côté et sortent par l'autre.

L'espace n'est pas présenté comme un réseau de communication ni de circulation, ni non plus comme un tissu urbain, mais comme une contingence obligatoire pour passer d'un lieu à un autre de manière intelligible. Comme dans les télé-séries ou les bandes dessinées québécoises, un plan de contextualisation vaut pour l'ensemble de la production. Malgré toutes les objections d'ordre matériel et financier, on doit constater qu'aucun autobus ne traverse jamais le champ, que les décors ont été vidés de leurs passants pour être remplacés par des grands-mères, des femmes enceintes, des femmes avec poussette aux côtés de figures grossièrement pittoresques (juifs Hassidim, orchestre municipal, etc). En regard de ce qui précède la conservation d'une anecdote frappe le spectateur. Il s'agit d'une scène devant l'hôtel où pénètre Florent. Au lieu de couper le plan pour entrer dans la bâtisse, la caméra reste sur place et continue de tourner. Elle donne ainsi à voir l'arrivée au carrefour d'une auto de luxe; deux passants tapent sur le capot et se font apostropher par le propriétaire du véhicule. Quand la rue n'est pas une contingence, elle est un lieu d'affrontement de classes dans un monde d'argent. Comment, à la suite de cela, ne pas remarquer le temps accordé à l'espace campagnard autour de Ste-Romanie, bien plus ouvert et bien moins agressant?

La télé-série, bien que trois fois plus longue que le film, n'offre pas de solution différente. Les séquences supplémentaires sont de l'ordre du renforcement: davantage de scènes avec les parents et avec la gardienne d'Émile, davantage de scènes sur les relations entre Florent et Élise, etc. L'épisode en Floride est entièrement centré autour du personnage de la tante et ne compte aucune ouverture sur un espace alternatif. Notons, d'ailleurs, l'absence dans les deux productions de l'abbé Jeunehomme.

Cette adaptation de Beaudin est une régression par rapport au roman et il faut peut-être penser que le public ne s'y est pas trompé. 


\section{Le Dossier de presse}

Conforme à sa vocation, la presse n'a pas laissé échapper l'aubaine qui se présentait. L'aventure du Matou dure depuis cinq ans et certains magazines lui consacrent encore des couvertures (TV plus, 31 janvier -6 février 87; Châtelaine, janvier 87).

J'ai dénombré trente-six périodiques, hebdomadaires, mensuels ou journaliers, québécois, anglophones ou européens qui ont traité du Matou de 1982 à 1987. Sans être exhaustif, ce dossier de la presse écrite laisse entendre suffisamment de voix pour que se mette en place après la saga d'Yves Beauchemin puis celle de Jean Beaudin, la saga des journalistes, troisième variation sur le thème «Florent veut un restaurant».

Rappelons que le livre est sorti à la fin de l'année 1981, dans la présentation courante des éditions Québec/Amérique. Grâce au succès de librairie, le roman est distribué en Europe dès 1982; au même moment, des traductions sont négociées. En 1985, sortent l'édition de poche et le film, l'affiche de l'un servant de couverture à l'autre; en 1987, est diffusée la télé-série sur le réseau de Radio-Canada. L'auteur a d'ores et déjà participé à des chroniques radiophoniques qui ont été rassemblées en un journal biographique à la fin de 1986. Enfin, il existe un disque sur lequel a été enregistré la musique du film.

Les articles recensés portent sur l'ensemble de ces produits et démontrent la remarquable longévité de l'opération commerciale. Elle est supportée par la vitalité d'un récit dont les journalistes définissent eux-mêmes les bornes.

Quant au Matou, il ne nous apparaît pas non plus comme une oeuvre de combat idéologique. C'est un fort roman picaresque (...) Le Matou pourrait aussi bien être un roman américain ou français: il est né de "la ville»-Montréal, New York ou Paris, peu importe - (...) Tout cela (...) n'a plus rien à voir avec les proses grinçantes, militantes des années soixante, et encore moins avec l'altière agressivité des textes théoriques d'alors... Le Matou me semble exemplaire de cette littérature décrispée que doit produire aujourd'hui la communauté francophone du Canada.

(François Nourissier, le Point, non daté)

Je le savais avant de partir que c'était un winner et ça me plaisait énormément (...). C'est ça qui est nouveau au Québec. De plus en plus, les Québécois prennent leurs affaires en mains. Finalement les gens commencent à avoir confiance. Ils veulent gagner, ils veulent réussir. Il faut arrêter de parler des losers.

(Jean Beaudin, La Presse, entrevue, 24 août 1985)

L' «effet-média» prend son essor lorsque le phénomène du best-seller se fait sentir. La critique qui s'occupe du roman en 1981 et 1982 est une critique conforme au modèle courant du compte rendu de lecture. Puis, selon la formule consacrée, "les médias s'en emparent» et construisent un univers référentiel dans lequel se place, à un endroit précis, le texte dont il est ques- 
tion. Ce faisant, les médias bâtissent la crédibilité du texte et lui attribuent sà mythographie particulière.

Tout d'abord, ils lui trouvent une place dans le Panthéon de l'Histoire littéraire. Par une profusion de comparaisons, l'auteur rivalise, entre autres, avec Balzac, Joyce, Dickens, Kafka, Vian, Colette, Rabelais, Queneau, accédant par là à quelques classes de genres littéraires (réalisme, fantastique, humour, etc.).

Puis, ils le situent dans le cadre de la culture contemporaine. De façon assez remarquable, l'œuvre n'est pas alors comparée à celle d'autres écrivains québécois ou étrangers, mais à celles de vedettes nationales des domaines de la chanson, de la peinture, de la politique (Diane Dufresne, Riopelle, Lemieux, Lise Payette ${ }^{4}$ ). Mentionnons une comparaison avec Serge Reggiani et une autre avec le corpus de la bande dessinée sans mention particulière d'auteur. Ce rétrécissement soudain de l'univers référentiel laisse entendre que la tradition littéraire puise ses références dans la vieille Europe - qu'on se rappelle ici le récit de l'abbé Jeunehomme - le territoire national offrant plutôt son espace à des vedettes.

Pour parachever le caractère culturel du texte, ils puisent dans l'institution littéraire le prestige des diplômes et cherchent par quelques incursions à établir si l'auteur est ou non licencié es lettres (les réponses à ce sujet sont contradictoires).

La solidité du produit en tant que valeur commerciale fournit davantage de données tangibles (tirage, état des ventes, droits pour la traduction et l'adaptation, première et deuxième édition), cet aspect permettant de préciser que des droits éventuels sont discutés avec l'URSS. "Même dans l'autre camp, on lit le Matou»: le texte prend des dimensions planétaires.

Enfin, ils vont chercher dans le passé de l'auteur les signes de sa prédestination, tant littéraire que cinématographique.

On peut encore signaler le texte d'une journaliste qui, fidèle en cela à l'idéologie du roman, fustige Pélagie-la Charette d'Antonine Maillet pour faire du Matou un texte supérieur à ceux des lauréats du prix Goncourt (L'Actualité, Février 83).

On est donc en présence d'une vedette d'exposition, dont la performance a revêtu la forme d'un texte écrit rapportant gros. Dans un même mouvement, cette performance marque d'une pierre blanche l'histoire littéraire; son auteur est meilleur que les autres, il est érudit et son destin est tracé depuis l'enfance. La jonction harmonieuse entre ces deux visions peut paraître difficilement concevable. Elle est pourtant déjà présente dans le roman sous la forme des deux récits articulés autour de Florent et de l'abbé Jeunehomme. En regard de ce qui précède, les déclarations faites par l'auteur lui-même font effet de rupture. Il exprime ce qu'il considère comme ses repères. Dans le domaine littéraire, il évoque Alphonse Allais, le modèle, mais aussi, l'anti-modèle Claude-Henri Grignon. Les médias n'ont pas repris à leur compte ces rensei- 
gnements. De fait, Alphonse Allais n'occupe qu'une modeste place auprès des grands de la littérature. L'éviction de Claude-Henri Grignon semble une occultation pure et simple. En effet, non seulement l'auteur en a-t-il parlé dans ses entrevues, mais encore trouve-t-on dans les premières pages du roman une comparaison explicite d'un personnage avec Séraphin (p. 20).

Dans ce dossier de presse, certains articles sont plus distanciés que d'autres (comme ceux du Devoir ou du Point contrairement à ceux de l'Actualité). Selon le public cible et la ligne rédactionnelle, chaque périodique privilégie un aspect ou un autre: de la visite de la Binerie à l'inventaire de la discographie d'Yves Beauchemin, en passant par le curriculum vitae du chat-acteur ou de Jean Carmet. Excroissance typique du phénomène média, la survalorisation de l'enfant-acteur, catalyseur des affects - et qui, lui, survit à son personnage: exorcisme? - qui, enfin à la sortie du film, vole la vedette à Florent/Serge Dupire. À poids égal avec le romancier lui-même, il fait la couverture de TV-plus en février 87 quand l'auteur a droit à une mention sans photographie en couverture de Châtelaine en janvier 87.

Désormais, Yves Beauchemin et le Matou appartiennent au domaine public et chacun se l'est approprié à son goût. Réginald Martel, comme à propos, fait alors une critique d'humeur de la biographie/journal ${ }^{5}$ de l'auteur:

C'était une belle histoire, une belle aventure dont on garde un souvenir pieux et sentimental.

(La Presse, 6 décembre 86)

Bientôt paraîtra le nouveau roman d'Yves Beauchemin et l'on pourra observer si un nouveau chapitre de la saga a lieu ou non. Il faut cependant garder à l'esprit que les médias ne font pas l'Histoire de leur propre initiative. En effet, ils avaient accordé au film, d'emblée, le crédit d'un succès similaire à celui du livre. La presse s'est vite tue devant le désintérêt du public ${ }^{6}$.

\section{Les couvertures et l'affiche}

Aux deux extrémités de cette chaîne de production, se trouvent deux images: celle de la couverture de la première édition et celle de l'édition de poche qui a aussi servi d'affiche au film. Dans l'une, nous sommes dans l'ordre du récit; un cadrage quadrangulaire ouvre sur un univers spécifique, construit et homogène. Obéissant aux règles classiques du réalisme, l'image représente un petit garçon, au bord d'une galerie, qui regarde vers un point du hors-champ diégétique.

Dans l'autre, nous sommes dans l'ordre du discours; un procédé de collages juxtapose des personnages de tailles diverses et nous laisse recevoir le regard direct de Florent et Élise. Malgré leur taille réduite, leur position stratégique et leur adresse au spectateur en font les principaux protagonistes de l'image. Iconographiquement, ils sont dans la veine de la romance «Harlequine». Ici, point d'univers englobant; chaque personnage a une taille en propre, est enfermé dans sa propre sphère et prend statut de figure fétiche. 
Ce changement d'illustration est représentatif de l'évolution de la chaîne des productions. Ces deux images en donnent une vision synthétique et concordent avec les constatations effectuées tout au long de notre analyse.

Les réflexions qui précèdent ne permettent pas de rendre compte intégralement des productions engendrées par le roman. Il serait possible encore de considérer la couverture faite par les médias audio-visuels, l'enregistrement sur disque, et donc l'interprétation de François Dompierre, les conférences commandées à l'auteur par les maisons de la culture et les institutions d'enseignement, etc.

Cependant, on peut dès à présent mesurer l'effet tentaculaire de l'opération et la manière dont elle s'est épanouie autour de nous. L'importance du Matou peut s'évaluer à la superficie qu'il occupe dans le champ culturel, dans un effet d'exposition. Reste à s'interroger sur la portée de cette opération: force est de constater que les enjeux idéologiques investis dans l'affaire sont passés de l'ordre de la réduplication et du conservatisme (Beauchemin), à celui de la réaction (Beaudin et la presse) pour être ensuite vidés de contenu (affiche et deuxième couverture) au profit de la pure valeur d'exposition (le «Regardez-nous» de Florent et Élise).

Il demeure pourtant que l'engouement pour le Matou se présente comme une manifestation d'intérêt pour un "gagnant» et que cela n'est peut-être pas étranger à la préparation de l'éclatante réception du Déclin de Denis Arcand: affaire à suivre.

1. Yves Beauchemin, le Matou, Montréal, Québec-Amérique, 1981, 583 p.

2. On reconnaîtra ici des propositions méthodologiques de Philippe Hamon telles qu'il les formule, par exemple, dans "Pour un statut sémiologique du personnage", in Poétique du récit, Paris, Seuil, coll. «Points» no 78, 1977, p. 115 et svtes.

3. Le Matou, Qc, réal. Jean Beaudin, $1985,180 \mathrm{mn}$.

Le Matou, Qc, réal. Jean Beaudin, télé-série en six épisodes, 1987, télévision de Radio-Canada.

4. L'Actualité, février 1983.

5. Yves Beauchemin, Du sommet d'un arbre, Montréal, Québec/Amérique, 1986, $140 \mathrm{p}$.

6. Cf. La Presse. 6 février 1986: "la version originale du Matou ne satisfait pas les Français» et le Nouvelliste de Trois-Rivières, 8 février 1986: "La carrière du Matou compromise en France». Après un record d'entrées au cinéma Berri, le film est très vite retiré de l'affiche. 


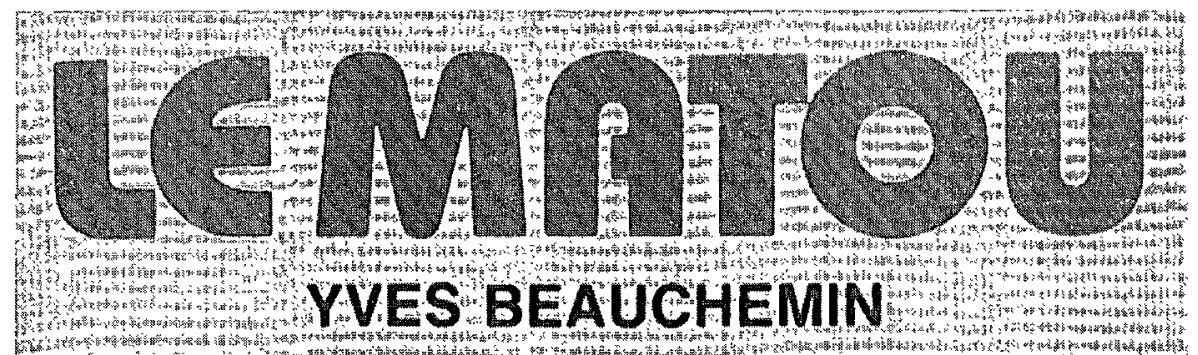

A A

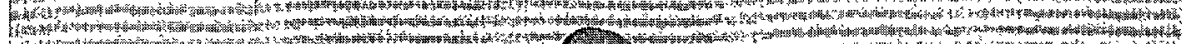

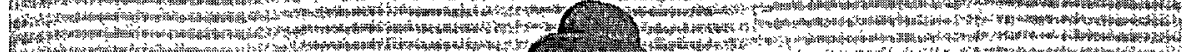

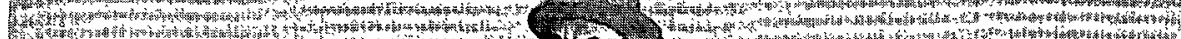

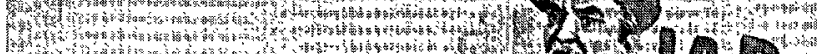

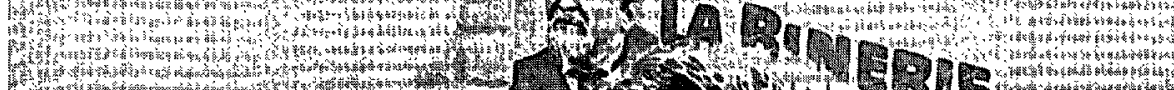

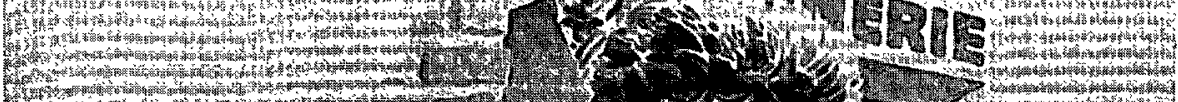
W W

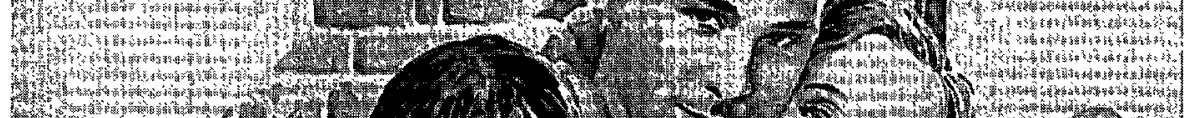

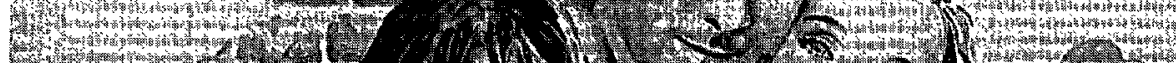
W. 1 कर

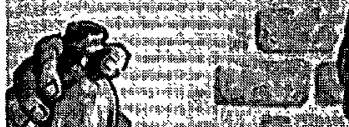
1.

.

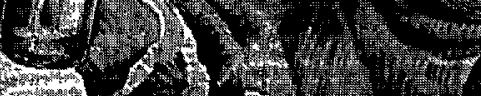

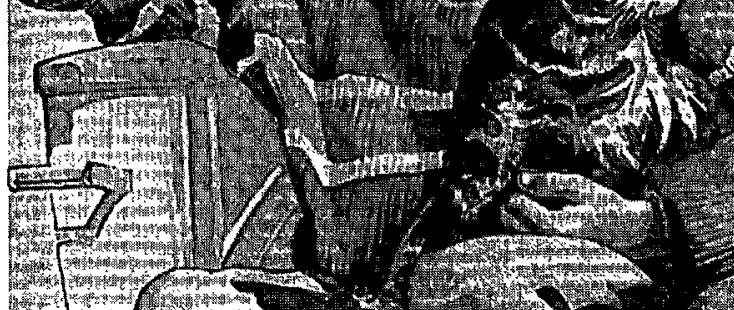

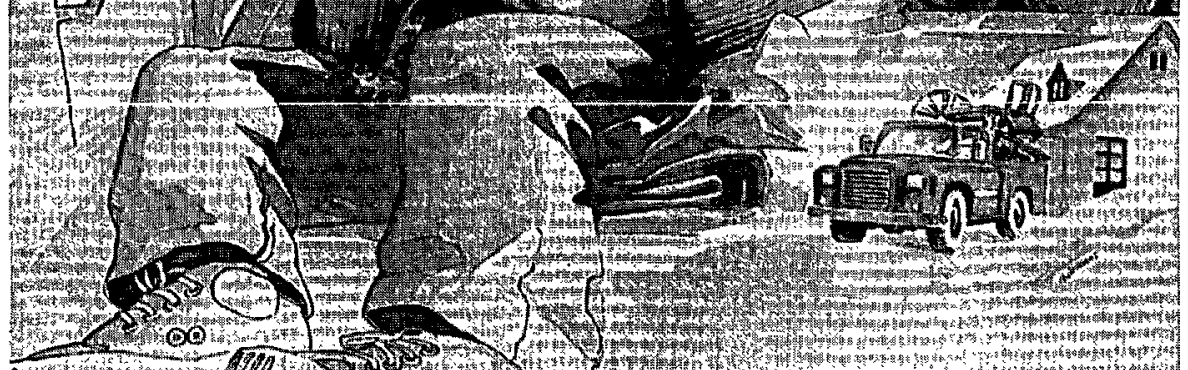

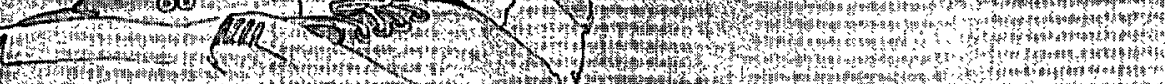
2.

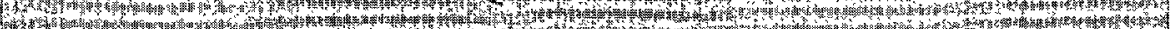

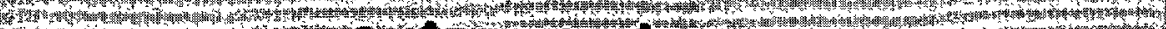
4tos

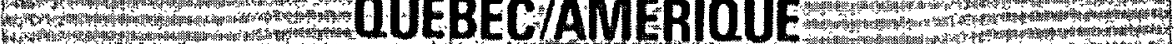

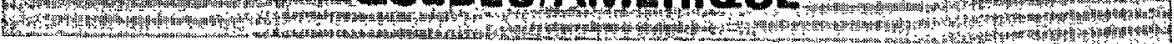

Édition de poche. 